\title{
Long Non-Coding RNA XLOC_008466 Functions as an Oncogene in Human Non- Small Cell Lung Cancer by Targeting miR- 874
}

\author{
Rui Yanga Ping Lia Guojun Zhang ${ }^{a}$ Chunya Lu ${ }^{a}$ Huaqi Wang ${ }^{a}$ Guoqiang Zhao ${ }^{b}$ \\ aDepartment of Respiratory Medicine, the First Affiliated Hospital, Zhengzhou University, Zhengzhou, \\ China, bepartment of Microbiology and Immunology, College of Basic Medical Sciences, Zhengzhou \\ University, Zhengzhou, China
}

\section{Key Words}

Non-small-cell lung cancers (NSCLC) • XLOC_008466 • miR-874 • MMP2 • XIAP

\begin{abstract}
Background: The therapy and prognosis of lung cancer are difficult because of multiple genetic and epigenetic alterations. Long non-coding RNAs (IncRNAs) have been verified as new mediators of cancer development and progression by virtue of their various functions. Here, we focused on the IncRNA XLOC_008466 based on previous microarray data. However, whether aberrant expression of XLOC_008466 in human non-small cell lung cancer (NSCLC) is correlated with malignancy, metastasis or prognosis has not been elucidated. Methods: We performed real-time PCR, CCK-8, flow cytometry, trans-well, western blotting, luciferase reporter assays, RNA immunoprecipitation (RIP) assay and surface plasmon resonance (SPR) assay to detect the function of XLOC_008466 in NSCLC. Results: Up-regulation of XLOC_008466 in NSCLC patients was related to lymph node metastasis and the TNM stage. In vitro, down-regulation of XLOC_008466 inhibited cell proliferation and invasion of A549 and $\mathrm{H} 460$ cells in vitro, but promoted cell apoptosis. Experiments on mechanisms revealed that XLOC_008466 functioned as a ceRNA, directly binding to miR-874, and could affect cell proliferation, apoptosis and invasion through regulation of miR-874 expression as well as by increasing matrix metalloproteinase 2 (MMP2) and X-linked inhibitor of apoptosis (XIAP) expression. Conclusions: XLOC_008466 functions as an oncogene in NSCLC by regulating the miR-874-MMP2/XIAP axis, which indicates that XLOC_008466 may be a useful marker and potential therapeutic target in NSCLC.

\section{Introduction}

The incidence of lung cancer has increased considerably in recent years, and it is one of the most prevalent and deadliest cancers in the world [1]. Non-small cell lung cancer

Guojun Zhang

and Guoqiang Zhao

KARGER
Dept Respiratory Medicine, the First Affiliated Hospital of Zhengzhou University, No. 40 Daxue Road, Zhengzhou 450052 (China) and Dept Microbiol Immunol, College of Basic Medical Sciences, Zhengzhou University, No.100 Kexue Road, Zhengzhou 450001 (China)

E-Mail gjzhangzzu@126.com / zhaogq@zzu.edu.cn 
(NSCLC) and small cell lung cancer are the two main histological types of lung cancer. The former, NSCLC, is the predominant form of lung cancer, accounting for approximately 80$85 \%$ of all incidences and has poor therapeutic efficacy when diagnosed [2]. Despite great improvements in treatment strategies, including surgery, chemotherapy, and radiation therapy, supplemented with targeted therapy and immunotherapy, the prognosis of these patients remains poor, with $10-15 \%$ having a 5-year survival [3-6]. The development of cancer involves multiple factors that may affect treatment and prognosis. Therefore, it is necessary to explore and analyze the relevant molecular mechanisms of lung cancer for limiting cancer progress or identifying potential therapeutic targets.

With the rapid development of bioinformatics analyses and application of nextgeneration sequencing technology to whole genomes and transcriptomes, non-coding RNAs (ncRNAs) are attracting the most attention among multiple factors involved in cancer development. ncRNAs are divided into two major groups based on their size, small ncRNAs and long noncoding RNAs (lncRNAs). Small ncRNAs include the more recently discovered and well-defined miRNAs and siRNAs $[7,8]$. IncRNAs are defined as non-protein coding transcripts longer than 200 nucleotides. In contrast to small ncRNAs, IncRNAs are not generally highly conserved. In recent years, IncRNAs have been identified as new mediators of tumorigenesis by virtue of their various functions and capacity to induce different mechanisms as a result of their wide spectrum of interactions [9-11]. Furthermore, several authors have proposed that there is interplay between IncRNAs and miRNAs during the tumorigenic process, from which competing endogenous RNAs (ceRNAs) are derived [12]. Additionally, miRNAs are involved in multiple biological processes by regulating their mRNA targets. It is possible that the lncRNA-miRNA-mRNA interaction network plays an important role in the development of cancer.

Based on our previous microarray data analysis of lncRNAs in NSCLC tissues [13], our present study was performed to confirm the expression of XLOC_008466 and explore the potential regulatory network of XLOC_008466-miRNA-mRNA using bioinformatics prediction and sequence analysis.

\section{Materials and Methods}

\section{Patients and samples}

Samples of human NSCLC tissue and matched adjacent normal tissue were obtained from 63 patients who underwent surgical resection between January 2014 and November 2015 at the First Affiliated Hospital of Zhengzhou University (Zhengzhou, China). Patients were diagnosed as NSCLC by histopathology combined with clinical information. All samples were immediately snap-frozen in liquid nitrogen until total RNA extraction. Clinicopathological parameters were correspondingly collected from databases at our institutions. All protocols were reviewed and approved by the Ethics Committee of Zhengzhou University, and all patients gave written informed consent.

\section{Cells culture}

Human NSCLC cell lines A549 and H460 and normal human bronchial epithelial cells (NHBE) and were obtained from the Type Culture Collection of the Chinese Academy of Sciences (Shanghai, China). HEK293T cells were a generous gift from Pro. Zhao (College of Basic Medical Sciences of Zhengzhou University, China). A549 and H460 cells were grown in RPMI-1640 medium (Gibco, USA) supplemented with 10\% FBS (Gibco, USA), $100 \mathrm{U} / \mathrm{mL}$ penicillin and $100 \mu \mathrm{g} / \mathrm{mL}$ streptomycin. NHBE cells were cultured with bronchial epithelial cell growth medium (BEGM, Gibco, USA) and the necessary supplements, and HEK-293T cells were maintained in DMEM medium (Gibco, USA). All cells were cultured in a $5 \% \mathrm{CO}_{2}$ humidified atmosphere at $37^{\circ} \mathrm{C}$, Medium was renewed every day, and cells were passaged every three days using $0.25 \%$ trypsin.

\section{Cell transfection and reagents}

For analysis of XLOC_008466, siRNA against XLOC_008466 (for the siRNA Lnc group) and negative control oligonucleotides (for NC group) were synthesized by Shanghai GenePharma Co. Ltd (Shanghai, 


\section{Cellular Physiology Cell Physiol Biochem 2017;42:126-136 \begin{tabular}{l|l} 
DOI: 10.1159/000477121 & and Biochemistry \\
Published online: May 15, 2017 & $\begin{array}{l}\text { 2017 The Author(s). Published by S. Karger AG, Basel } \\
\text { www.karger.com/cpb }\end{array}$
\end{tabular}}

Yang et al.: XLOC_008466 Functions as an Oncogene in NSCLC

China). For analysis of XLOC_008466 related miRNA, a miR-874 mimic (for the miR-874 group) and scrambled oligonucleotides (for the miR-NC group) were also synthesized by Shanghai GenePharma Co. Ltd. For further analysis, the recombinant plasmids pmirGLO-XLOC_008466-wt/pmirGLO- XLOC_008466mut and pmirGLO-XIAP-wt/pmirGLO-XIAP-mut were prepared. For transfection, Lipofectamine ${ }^{\mathrm{TM}} 2000$ (Invitrogen, USA) was used as the transfection reagent according to the manufacturer's instructions.

\section{RNA extraction and quantitative real-time PCR ( $q R T-P C R$ )}

In tissue samples and different cell groups, total RNA was extracted using TRIzol reagent (Invitrogen, USA), according to the manufacturer's instructions. The RNA quality and concentration were assessed with a NanoDrop 1000 Spectrophotometer (Thermo Fisher Scientific, USA). To verify the relative expression of XLOC_008466, MMP2 and XIAP, reverse transcription was performed using a First Strand cDNA Synthesis Kit (Qiagen, Germany). Then, the ABI SYBR ${ }^{\circledR}$ Green PCR Master Mix (Applied Biosystems, USA) was used for quantitative real-time PCR, with GAPDH as the reference gene. To verify the relative expression of miR874, qRT-PCR was performed using a miR-874 qRT-PCR Detection Kit (Stratagene Corp, USA) in conjunction with an ABI 7500 thermal cycler. U6 small nuclear RNA (U6 snRNA) was used as an endogenous control for normalization. In addition, melting curves were used to evaluate non-specific amplification. The qRT-PCR results are expressed as the threshold cycle $(\mathrm{Ct})$ and were converted to fold changes $\left(2^{-\Delta \Delta t}\right.$ or $\left.2^{-\Delta c t}\right)$.

\section{Protein extraction and western blotting}

In different cell groups, total proteins were extracted and subjected to $10 \%$ sodium dodecyl sulfate polyacrylamide gel electrophoresis for separation and then transferred onto polyvinylidene difluoride membranes (Whatman, UK). Blocking was performed in 5\% skim milk for $1 \mathrm{~h}$. Then, membranes were washed four times with wash buffer at room temperature and incubated overnight at $4^{\circ} \mathrm{C}$ with primary antibodies (Santa Cruz, USA). In this study, rabbit anti-human matrix metalloproteinase 2 (MMP2) (1:500) and rabbit anti-human X-linked inhibitor of apoptosis (XIAP) (1:500) were used. The secondary antibody (goat anti-rabbit IgG, 1:1000, Santa Cruz, USA) was incubated for $1 \mathrm{~h}$. After washing, signals from different proteins were determined using a chemiluminescence detection kit (Amersham Pharmacia Biotech, UK). GAPDH (Santa Cruz, USA) served as an endogenous control.

\section{Cell proliferation assay}

A cell proliferation assay was performed using Cell Counting Kit-8 solution (Dojindo, Japan) in accordance with the manufacturer's protocol. Briefly, A549 and H460 cells in different groups were seeded into 96-well plates at a concentration of $2 \times 10^{3}$ cells/well. Each group had three replicate wells. According to the experimental requirements, we observed cell proliferation at $24 \mathrm{~h}, 48 \mathrm{~h}$ and $72 \mathrm{~h}$. Before observation, each well was treated with $10 \mu \mathrm{L} /$ well of cell counting kit-8 solution during the last $4 \mathrm{~h}$ of culture. Then, cellular viability was determined by measuring the absorbance of the converted dye at $450 \mathrm{~nm}$.

\section{Cell apoptosis assay}

A cell apoptosis assay was performed using flow cytometry with propidium iodide (PI) and Annexin $\mathrm{V}$ double staining (BestBio, China). Specifically, A549 and H460 cells in different groups were harvested by trypsinization and resuspended at a density of $1 \times 10^{6}$ cells $/ \mathrm{mL}$. Annexin V-fluorescein isothiocyanate (FITC) and PI were used for double staining to assess the proportions of apoptotic cells. Data were read with a FACScan flow cytometer (BD Biosciences, USA).

\section{Cell invasion assay}

A cell invasion assay was performed using the trans-well invasion method. Specifically, A549 and H460 cells in different groups were seeded into the upper chamber of a 24-well Tran-swell Permeable Support (Costar, USA) at a concentration of $2 \times 10^{5}$ cells/well. The lower chamber was supplied with $500 \mu \mathrm{L}$ of medium containing $10 \%$ FBS. The 24 -well plates were incubated at $37^{\circ} \mathrm{C} / 5 \% \mathrm{CO}_{2}$ for $48 \mathrm{~h}$. The medium was removed from the upper chamber and cells in the upper chamber were scraped off with a cotton swab. Cells that had moved to the other side of the membrane were fixed with methanol, stained with hematoxylin, mounted, and dried at $80^{\circ} \mathrm{C}$ for $30 \mathrm{~min}$. The number of cells invading the matrigel was counted in three randomly selected fields using an inverted microscope. 


\section{Cellular Physiology Cell Physiol Biochem 2017;42:126-136 \begin{tabular}{l|l} 
DOI: 10.1159/000477121 & O 2017 The Author(s). Published by S. Karger AG, Basel \\
www.karger.com/cpb
\end{tabular}}

Yang et al.: XLOC_008466 Functions as an Oncogene in NSCLC

\section{Luciferase reporter assay}

To further explore the relative mechanism of XLOC_008466 function in NSCLC, we predicted the target genes of XLOC_008466 using a bioinformatics prediction (miRcode and TargetScan program) combined with sequence analysis. Based on the predicted results and preliminary experiments, we constructed the recombinant plasmid pmirGLO-XLOC_008466-wt/pmirGLO-XLOC_008466-mut, pmirGLO-MMP2-wt/ pmirGLO-MMP2-mut and pmirGLO-XIAP-wt/pmirGLO-XIAP

-mut for further investigation. PCR was used to obtain the wild-type XLOC_008466 sequence (XLOC_008466-wt) and wild-type 3'UTR of MMP2 and XIAP mRNA. A mutant XLOC_008466 (XLOC_008466mut) without miR-874 binding sites and mutant MMP2 and XIAP 3'UTR fragments was obtained by overlap extension PCR with the mutant primers. For the luciferase reporter assay, the recombinant plasmids were transiently co-transfected with miR-874 mimics or siRNA Lnc into HEK293T cells using Lipofectamine ${ }^{\mathrm{TM}} 2000$. Luciferase activity was measured $24 \mathrm{~h}$ post-transfection with the Dual Luciferase Reporter Assay System (Promega, USA) according to the manufacturer's instructions.

\section{RNA immunoprecipitation (RIP) assay}

The RIP assay is an antibody-based technique that is used to map RNA-protein interactions in vivo. To further verify the relationship between XLOC_008466 and miR-874, we performed RIP using an EZMagna RIP RNA-binding protein immunoprecipitation kit (Millipore, USA), according to the manufacturer's instructions. A549 and $\mathrm{H} 460$ cells in different groups were lysed using RNA lysis buffer containing protease inhibitor and RNase inhibitor. Different cell lysis solutions were incubated with the RIP buffer containing magnetic beads coated with anti-human argonaute 2 (Ago2) antibodies (Millipore, USA). IgG (Millipore, USA) was used as a negative control (input group). Following incubation with gentle rotation for $2 \mathrm{~h}$ at $4^{\circ} \mathrm{C}$ and removal of the unbound material by washing, coprecipitated RNAs were isolated. Next, reverse transcription and PCR analysis were performed.

\section{Surface plasmon resonance (SPR) assay}

SPR assay was performed on a Biacore 3000 (BIAcore AB, GE Healthcare) using a carboxymethylated dextran coated sensor chip (CM5) at $25^{\circ} \mathrm{C}$. HBS-EP buffer was used as running buffer contained $10 \mathrm{mM}$ HEPES, $150 \mathrm{mM} \mathrm{NaCl}, 3.4 \mathrm{mM}$ EDTA and $0.005 \%$ P20 at pH 7.4. Biotin-labeled single-stranded RNA harboring the wild-type XLOC_008466 (5'-CTATGAGTCTGTGAGTTGCTTCAGGGCAG-3') and mutant sequence (5'-CTATGAGTCTGTGAGTTGCTTGTCCCGTC-3') were captured on sensor chip surface respectively, leaving another two empty channels as reference. miR-874 or scrambled oligonucleotides of different concentrations $(325.5 \mathrm{nM}, 625 \mathrm{nM}, 1250 \mathrm{nM}, 2500 \mathrm{nM}, 5000 \mathrm{nM}, 1000 \mathrm{nM}, 325.5 \mathrm{nM})$ were injected at $10 \mu \mathrm{L} /$ $\mathrm{min}$ across all the surfaces for $2 \mathrm{~min}$, and was then allowed to dissociate for $15 \mathrm{~min}$. All sensorgrams were evaluated by BIA evaluation software.

\section{Statistical analysis}

SPSS17.0 software was used for all statistical analyses. All of the results are expressed as the mean \pm SD. Student's t-test and a one-way analysis of variance (ANOVA) were used for the comparison of means from different samples. Pearson's correlation analysis was used to detect the correlation between XLOC_008466 and miR-874 expression. $P<0.05$ was considered to be statistically significant.

\section{Results}

XLOC_008466 is up-regulated in NSCLC patients

Our microarray data analysis revealed that the XLOC_008466 level was up-regulated in NSCLC tissues [13]. In this study, we further verified the expression of XLOC_008466 in 63 NSCLC patients and analyzed its expression features in the patient clinicopathological characteristics. qRT-PCR was performed, and the clinicopathological characteristics mainly included age, gender, smoking, tumor differentiation, TNM stage and lymph node metastasis. The results showed that XLOC_008466 was significantly up-regulated in NSCLC tissues compared to matched adjacent normal tissues (Fig. 1A, $P<0.001$ ). We also observed that XLOC_008466 expression was significantly related to lymph node metastasis and the TNM 
stage and was higher in the lymph node positive group or TNM III stage than the negative group or TNM I and II stage (Fig. 1B and 1C, $P<0.01$ ). Specifically, the other characteristics of the analyses are presented in Table 1.

Effects of XLOC_008466 down-regulation on cell proliferation, apoptosis and invasion

To synthesize XLOC_008466 expression and clinicopathological characteristics, bioinformatics analysis and our pre-experimentresults, we determined whetherXLOC_008466 had effects on the cell biology behaviors. A siRNA against XLOC_008466 was designed and transfected into A549 and H460 cells. The qRT-PCR results revealed that the XLOC_008466 levels were effectively reduced in the siRNA Lnc group for NHBE cells compared to the blank and NC groups (Fig. 2A, $P<0.01$ ). The cell proliferation assay showed that down-regulation of XLOC_008466 in the siRNA Lnc group led to slower proliferation $24 \mathrm{~h}$ after transfection than the blank group or scramble group, and the proliferation-suppressing trend became more obvious $48-72 \mathrm{~h}$ after transfection (Fig. 2B, 2C, $P<0.05$ ). Next, flow cytometry with PI and Annexin V double staining demonstrated that the apoptotic population was notably up-regulated in the siRNA Lnc group cells (approximately 14\% vs. 4\% in the control groups) (Fig. 2D, $P<0.01$ ). Moreover, we performed a trans-well invasion assay to assess the effect of XLOC_008466 on cell invasion. As expected, down-regulation of XLOC_008466 inhibited cell invasion (Fig. 2E, $P<0.01$ ). Together, these findings indicate that down-regulation of XLOC_008466 inhibits cell proliferation and invasion of A549 and H460 cells in vitro, but promotes cell apoptosis.

XLOC_008466 functions as ceRNA directly binding to miR-874

As stated in the introduction, increasing numbers of publications have demonstrated that lncRNAs may act as a ceRNA in regulating the biological functions of miRNA. We propose that XLOC_008466 functions as a ceRNA to regulate some miRNAs. Sequence analysis and a website program provided the putative binding sites between XLOC_008466 and miR-874 (Fig. 3A). To further test this hypothesis, we performed SPR assay to monitor XLOC_008466: miR874 interactions. The results showed a

Table 1. Association of XLOC_008466 expression with clinicopathological characteristics of NSCLC patients. ${ }^{*}$ Indicated statistical significance $(P<0.05)$

\begin{tabular}{|c|c|c|c|}
\hline \multirow{2}{*}{ Clinicopathological factors } & \multirow{2}{*}{$\mathrm{n}$} & \multicolumn{2}{|c|}{ XLOC_008466 expression ( $2-\Delta \Delta C t)$} \\
\hline & & Median \pm SD & $P$ \\
\hline \multicolumn{4}{|l|}{ Gender } \\
\hline Male & 40 & $3.7087 \pm 0.9570$ & 0.649 \\
\hline Female & 23 & $3.5948 \pm 0.9464$ & \\
\hline \multicolumn{4}{|l|}{ Age(years) } \\
\hline$\geq 60$ & 40 & $3.6150 \pm 0.9484$ & 0.569 \\
\hline$<60$ & 23 & $3.7578 \pm 0.9484$ & \\
\hline \multicolumn{4}{|l|}{ Smoking status } \\
\hline Smoking & 36 & $3.7888 \pm 1.0371$ & 0.242 \\
\hline No smoking & 27 & $3.5049 \pm 0.8019$ & \\
\hline \multicolumn{4}{|l|}{ Differentiation } \\
\hline Well & 32 & $3.7094 \pm 1.0152$ & 0.722 \\
\hline Moderate-Poor & 31 & $3.6235 \pm 0.8859$ & \\
\hline \multicolumn{4}{|l|}{ Lymphnode metastasis } \\
\hline Negative & 21 & $3.1871 \pm 0.9717$ & $0.004^{*}$ \\
\hline Positive & 42 & $3.9071 \pm 0.8478$ & \\
\hline \multicolumn{4}{|l|}{ TNM } \\
\hline $\mathrm{I}+\mathrm{II}$ & 41 & $3.4127 \pm 0.8850$ & $0.003^{*}$ \\
\hline III & 22 & $4.1413 \pm 0.8913$ & \\
\hline
\end{tabular}
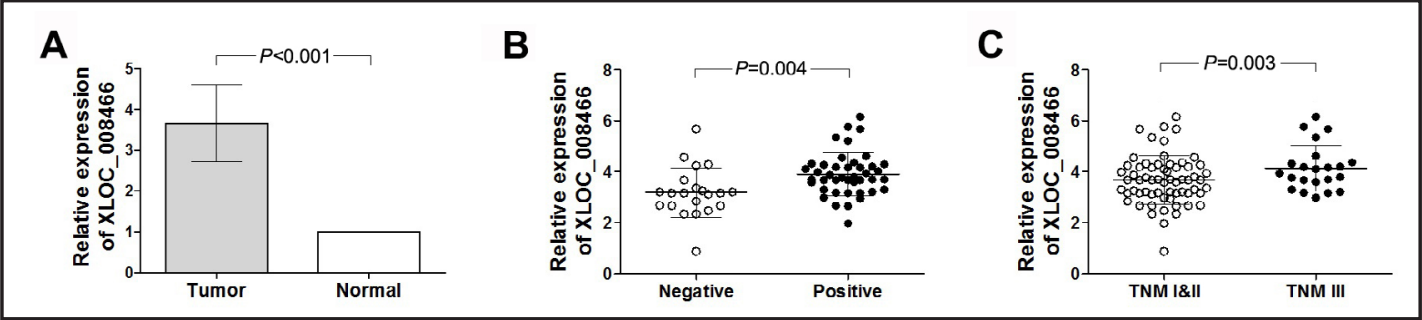

Fig. 1. Expression of XLOC_008466 in NSCLC patients. A: Up-regulation of XLOC_008466 in NSCLC tissues compared to matched adjacent normal tissues $(P<0.001)$. Data are presented as the mean \pm SD. B: Higher expression of XLOC_008466 in the lymph node positive group than the negative group $(P<0.01)$. C: Higher expression of XLOC_008466 in TNM III stage than TNM I and II stage $(P<0.01)$. The experiment was independently repeated in triplicate. 


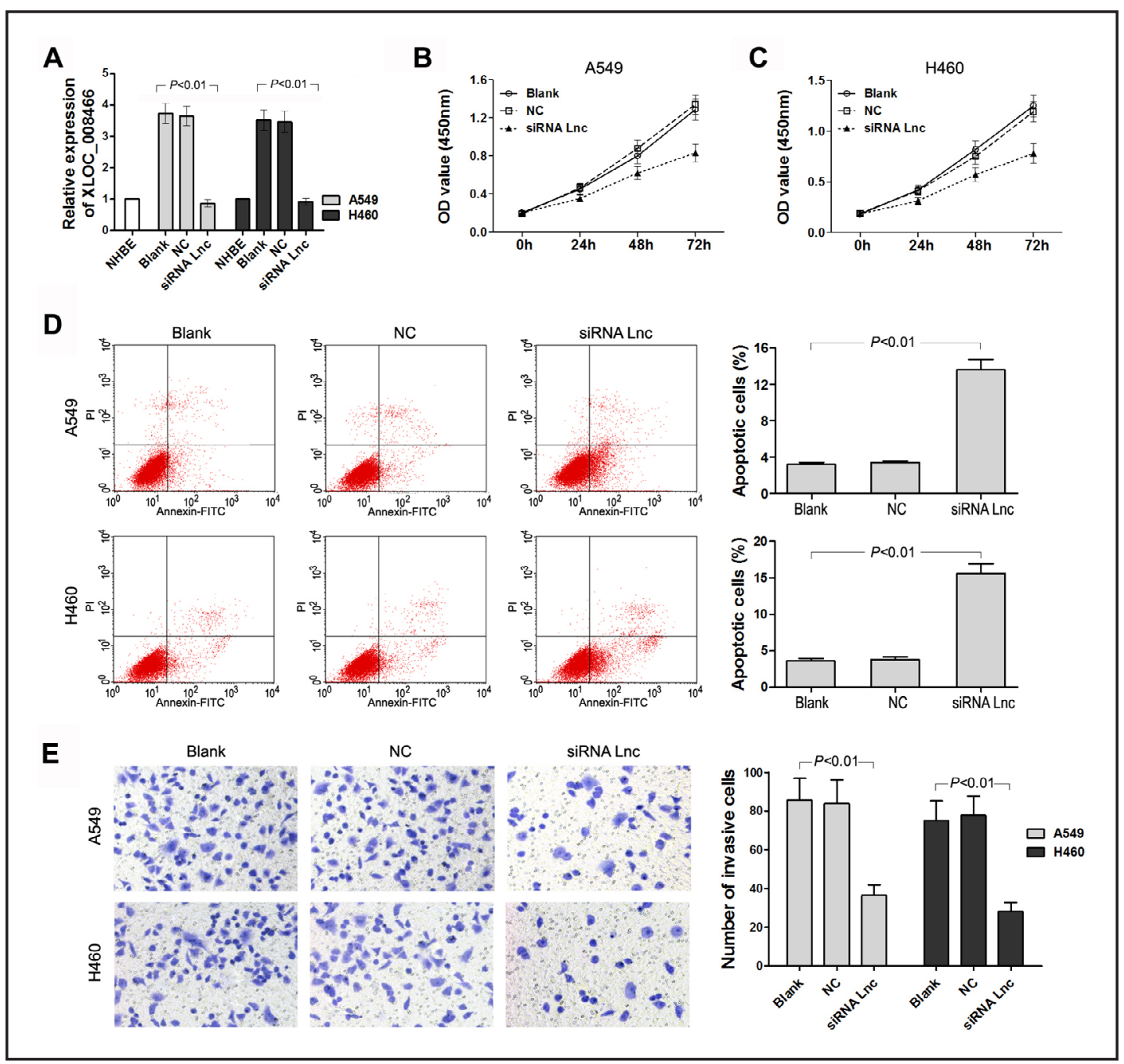

Fig. 2. Effects of XLOC_008466 down-regulation on cell proliferation, apoptosis and invasion. A: qRT-PCR shows effective down-regulation of XLOC_008466 in the siRNA Lnc group for NHBE cells compared to the blank and NC groups $(P<0.01)$. B, C: CCK-8 detection of the OD450 values in the siRNA Lnc group at $24 \mathrm{~h}$, $48 \mathrm{~h}$ and $72 \mathrm{~h}$ showing significant decreases in both A549 and H460 cells $(P<0.05)$. D: Flow cytometry demonstrated up-regulation of an apoptotic population in the siRNA Lnc group cells compared to the blank and NC groups $(P<0.01)$. E: Cell invasion of both A549 and H460 cells were significantly inhibited in siRNA Lnc group $(P<0.01)$. All experiments were independently repeated in triplicate.

positive binding between wild-type XLOC_008466 and miR-874 with dissociation constants $\left(\mathrm{K}_{\mathrm{D}}\right) 2.922 \pm 0.906 \mu \mathrm{M}$ (Fig.3B, $P<0.05$ ), which had statistical difference compared with other three control groups (Fig.3C, 3D, 3E). We also proceeded to use luciferase reporter assays to investigate the potential interaction of miRNAs with XLOC_008466. We obtained XLOC_008466-wt/XLOC_008466-mut through PCR and a mutagenesis kit. The fragments, including the predicted binding sites, were cloned into a pmirGLO vector as pmirGLOXLOC_008466-wt/ pmirGLO-XLOC_008466-mut. And then the recombinant plasmids were transiently co-transfected with a miR-874 mimic and scrambled oligonucleotides into HEK $293 \mathrm{~T}$ cells using Lipofectamine ${ }^{\mathrm{TM}} 2000$. Luciferase activities were measured $24 \mathrm{~h}$ posttransfection, and the results showed that the miR-874 mimic reduced the luciferase activity of pmirGLO-XLOC_008466-wt but not of pmirGLO-XLOC_008466-mut (Fig. 3F, $P<0.01$ ). Next, a RIP assay was performed using the Ago2 antibody. The qRT-PCR results showed that XLOC_008466 and miR-874 were preferentially enriched in Ago2-containing beads 


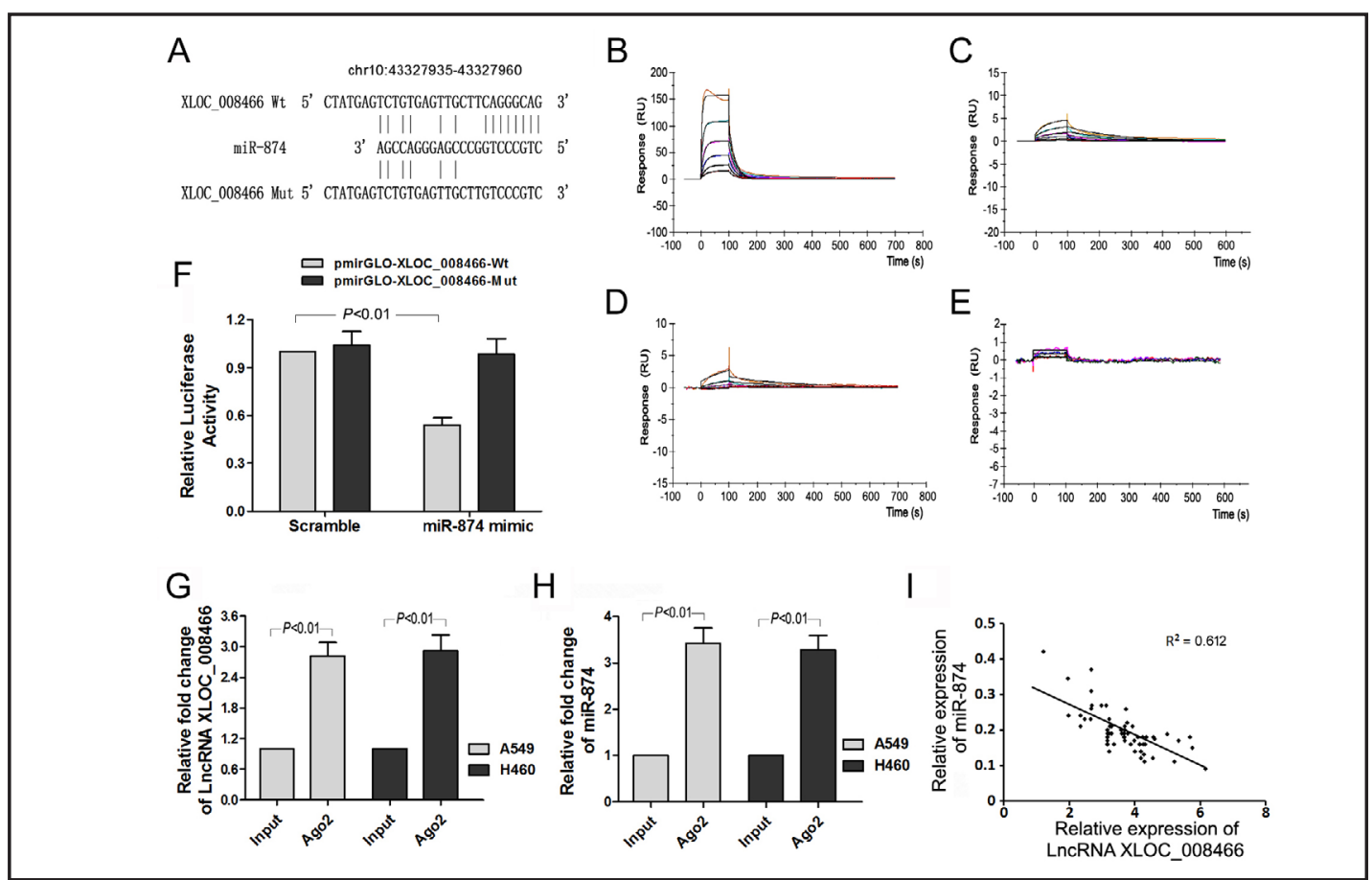

Fig. 3.XLOC_008466 functions as ceRNA directly binding to miR-874. A: The putative binding sites between XLOC_008466 and miR-874 and XLOC_008466-mut sequences. B: Hybridization between sensor-chips to which wild-type XLOC_008466 and injected miR-874 mimic (KD: 2.922 $\pm 0.906 \mu \mathrm{M})(P<0.05)$. C:Hybridization between sensor-chips to which mutantXLOC_008466 and injected miR-874 mimic (KD: $6.66 \pm 1.099 \mu \mathrm{M}$ ). D: Hybridization between sensor-chips to which wild-type XLOC_008466 and injected scrambled oligonucleotides (KD: $4.357 \pm 1.144 \mu \mathrm{M}$ ). E: Hybridization between sensor-chips to which mutant XLOC_008466 and injected scrambled oligonucleotides (KD: $824 \pm 2.171 \mu \mathrm{M})$. F: After 24 h post-transfection, the relative luciferase activities were reduced. G: RIP assay shows that XLOC_008466 is enriched in the Ago2-containing beads $(P<0.01)$. H: miR-874 is also enriched in the Ago2-containing beads $(P<0.01)$. I: The inverse relationship between XLOC_008466 and miR-874 expression $\left(\mathrm{R}^{2}=0.612, P<0.05\right)$. All experiments were independently repeated in triplicate.

compared to the input group (Fig. 3G, 3H, $P<0.01$ ). Combined with the inverse relationship between XLOC_008466 and miR-874 expression (Fig. 3I, $\mathrm{R}^{2}=0.612, P<0.05$ ). These data led us to elucidate that XLOC_008466 functions as a ceRNA, interacting with miR-874.

XLOC_008466 improves MMP2 and XIAP expression by down-regulating the miR-874 level

A bioinformatics analysis using TargetScan, microRNA.org, and miRanda indicated that MMP2 and XIAP were potential targets of miR-874 (Fig. 4A, 4B). Based on the data in Figure 3, we hypothesized that XLOC_008466 affects MMP2 and XIAP expression through down-regulation of the miR-874 level. pmirGLO-MMP2-wt or pmirGLO-MMP2-mut were cotransfected with the miR-874 mimic, scrambled oligonucleotides or siRNA for XLOC_008466 into HEK 293T cells using Lipofectamine ${ }^{\text {TM }} 2000$. Then, the luciferase activities were measured and showed that the miR-874 mimic reduced the luciferase activity of pmirGLOMMP2-wt, but not of pmirGLO-MMP2-mut, and the down-regulation of XLOC_008466 had the same effect (Fig. 4C, $P<0.05$ ). Similar results were obtained when the recombinant plasmids were pmirGLO-XIAP-wt/pmirGLO-XIAP-mut (Fig. 4D, $P<0.05$ ). We also observed the up-regulation of miR-874, down-regulation of MMP2 and XIAP mRNA in the siRNA Lnc group cells (Fig. 4E, 4F, 4G, $P<0.05$ ). Therefore, these data indicate that MMP2 and XIAP are targets of miR-874 and that XLOC_008466 can down-regulate miR-874 expression, as well as elevate MMP2 and XIAP expression.

\section{KARGER}


A

A xloc_008466 5' CTAtgagtctgtgagttgcttcaggGcag 3 ,

||||||||||||||
miR-874

Wt MMP2 3' UTR 5, CACAGGGCAGTGGGACAGGGCAT 3 ,

Mut MMP2 3' UTR 5 ' CACAGGGCAGTGGGAGTCCCGTT 3 ,

C

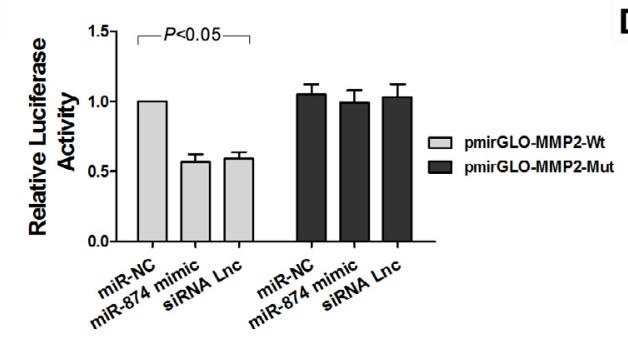

E
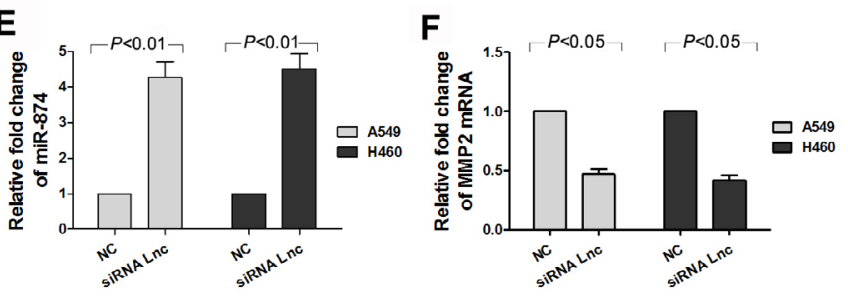

B

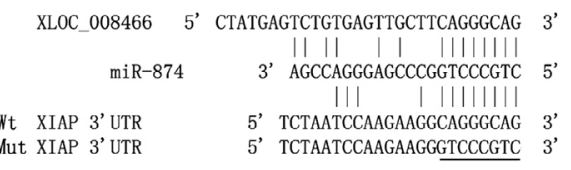

D

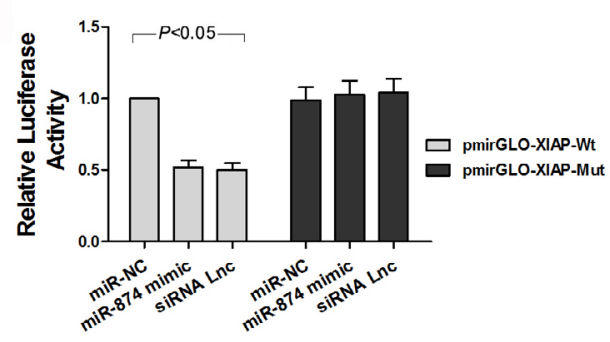

Fig. 4. XLOC_008466 improves MMP2 and XIAP expression by down-regulating the miR-874 level. A, B: The predicted target sites for miR-874 in the MMP2 and XIAP mRNA 3'UTR, and MMP2 and XIAP-mut sequences. C, D: After $24 \mathrm{~h}$ post-transfection, the relative luciferase activities were reduced in the miR-874 mimic and siRNA Lnc groups compared to the control group $(P<0.05)$. E: siRNA for XLOC_008466 significantly up-regulates miR-874 expression $(P<0.05)$. F, G: siRNA for XLOC_008466 significantly down-regulates MMP2 and XIAP mRNA expression compared to the control group $(P<0.05)$. All experiments were independently repeated in triplicate.

XLOC_008466 affects cell proliferation, apoptosis and invasion by regulating miR-874 expression and elevating MMP2 and XIAP expression

Based on the above results, we better understood the association among XLOC_008466, miR-874 and MMP2/XIAP. A series of experiments was performed to verify thatXLOC_008466 affected cell proliferation, apoptosis and invasion by regulating miR-874 expression and increasing MMP2 and XIAP expression in A549 and H460 cells. Cells were transfected with miR-874 mimic and siRNA for XLOC_008466, and down-regulation of MMP2 and XIAP expression was observed in the miR-874 group and siRNA Lnc group cells (Fig. 5A). In a CCK-8 assay, cells transfected with miR-874 showed a trend of lower proliferation rates, which was similar to the siRNA of XLOC_008466 transfected cells (Fig. 5B, 5C, $P<0.05$ ). A flow cytometry assay indicated that the cell apoptosis rate in the miR-874 group and siRNA Lnc group was higher than that of the control group (Fig. 5D, $P<0.05$ ). The trans-well invasion assay revealed that the cell invasion of the miR-874 group and siRNA Lnc group was significant decreased compared to that of the control group (Fig. $5 \mathrm{E}, P<0.05$ ). This series of results indicated that XLOC_008466 affects cell proliferation, apoptosis and invasion by regulating miR-874 expression and elevating MMP2 and XIAP expression, which suggests that XLOC_008466 functions as a ceRNA by directly binding to miR-874. It then affects more than one target mRNA and may participate in various signaling pathways.

\section{Discussion}

Cancer is a major cause of human disease-related death worldwide. Cancer biology is always regarded as a research hotspot. Emerging evidence suggests that lncRNAs act 
A

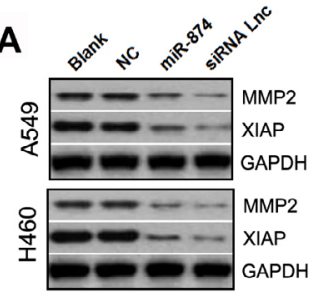

B

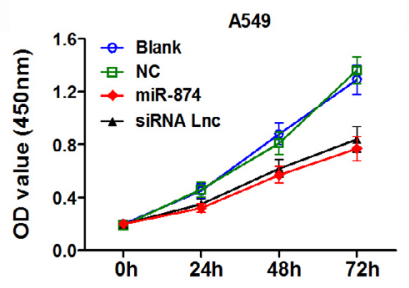

C

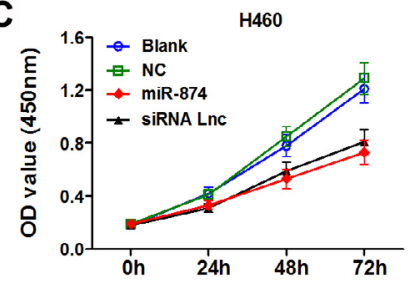

D

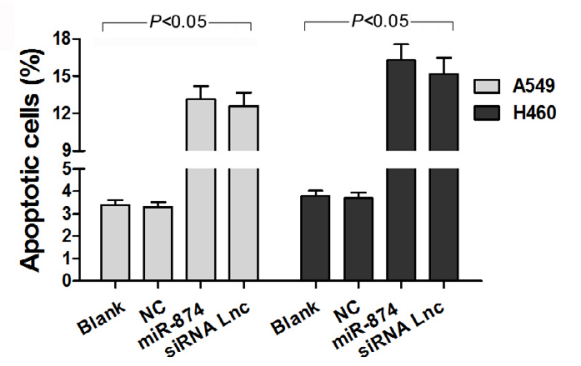

E

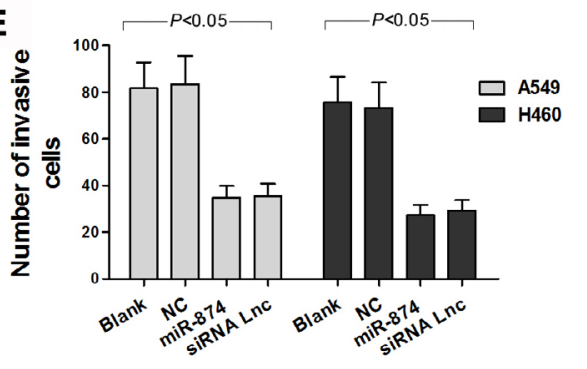

Fig. 5. XLOC_008466 affects cell proliferation, apoptosis and invasion by regulating miR-874 expression and elevating MMP2 and XIAP expression. A: Western blotting shows down-regulation of MMP2 and XIAP expression in the miR-874 group and siRNA Lnc group cells. B, C: The CCK-8 assay shows the lower proliferation rate in the miR-874 group and siRNA Lnc group cells $(P<0.05)$. D: The flow cytometry assay shows a higher apoptosis rate in the miR-874 group and miRNA Lnc group cells $(P<0.05)$. E: The trans-well invasion assay showed the inhibition of cell invasion in the miR-874 group and siRNA Lnc group $(P<0.05)$. All experiments were independently repeated in triplicate.

as drivers of tumor suppressive and oncogenic functions and constitute an important component of cancer biology. For example, MALAT1 is a novel cancer-associated lncRNA, and it has been shown to play an important role in gastric cancer, colorectal cancer, and more recently, lung cancer [14-21]. HOTAIR has been extensively studied in cancer [22]. Its expression has been shown to regulate breast and lung cancer cell growth and metastasis $[23,24]$. In our study, up-regulation of XLOC_008466 was confirmed in NSCLC tissues and is associated with lymph node metastasis and the TNM stage. Expression of XLOC_008466 in NSCLC patients with positive lymph nodes or TNM III stage showed more up-regulation than the negative group or TNM I and II stage, which suggests that XLOC_008466 expression is involved in the progression of NSCLC. In A549 and H460 cells, siRNA against XLOC_008466 was designed and effectively down-regulated XLOC_008466 expression. Down-regulation of XLOC_008466 inhibited cell proliferation, promoted cell apoptosis and impeded cell invasion. These results indicate that XLOC_008466 is a positive regulator of NSCLC development and therefore that XLOC_008466 down-expression may contribute to tumor progression.

The ceRNA hypothesis is gaining attention. This hypothesis is supported by a series of studies. In breast cancer, H19 encodes miR-675, and the link between H19/miR-675, Cbl and tyrosine kinase receptors enhances breast cancer cell aggressiveness [25]. Recent studies of MALAT1 in lung cancer revealed that MALAT1 could function as a ceRNA to regulate the expression of SLUG through competition for miR-204 [16]. HOTAIR also competitively binds to miR-193a in AML cells and functions as a ceRNA to modulate c-KIT (proto-oncogene) expression [26]. Therefore, the systematic identification of IncRNAs and their expression patterns in human tissues and disease is important for clarifying the molecular biology underlying cancer. Based on a bioinformatics prediction and sequence analysis, we found there was an interaction between XLOC_008466 and miR-874. In recent studies on miR-874, it was reported that miR-874 is down-regulated during NSCLC progression and suggested that the frequent loss of miR-874 leads to increased tumor malignancy [27]. In our study, we constructed pmirGLO-XLOC_008466-wt/pmirGLO-XLOC_008466-mut and transfected these 
plasmids with a miR-874 mimic into HEK 293T cells. The luciferase reporter assay confirmed that XLOC_008466 and miR-874 had a pairing region. The RIP assay and correlation analysis further suggested that XLOC_008466 functioned as a ceRNA by directly binding to miR-874. Not only that, we found that XLOC_008466 bound to miR-874 by SPR assay, confirming the findings of luciferase reporter and RIP assay.

Increasing evidence suggests that ceRNAs always affects the distribution of miRNAs to their targets and imposes an additional level of post-transcriptional regulation. We subsequently explored whether XLOC_008466 affects the targets of miR-874. Bioinformatics analysis indicated that MMP2 and XIAP are potential targets of miR-874. MMP2 plays an important role during the invasion and metastasis of various cancers, including NSCLC [28]. XIAP can regulate the apoptosis related pathway and induces cell apoptosis in NSCLC [29]. We also constructed pmirGLO-MMP2-wt/pmirGLO-MMP2-mut and pmirGLO-XIAPwt/ pmirGLO-XIAP-mut and transfected these with a miR-874 mimic or siRNA against XLOC_008466 into HEK 293T cells. The luciferase activities and qRT-PCR results indicated that XLOC_008466 down-regulated miR-874 expression and increased MMP2 and XIAP expression. Furthermore, a series of experiments verified that XLOC_008466 affects cell proliferation, apoptosis and invasion by binding to miR-874 to regulate MMP2 and XIAP expression. The results suggested that XLOC_008466 functions as a ceRNA by directly binding to miR-874 to regulate MMP2 and XIAP expression, which affects cell proliferation, promotes cell apoptosis and impedes cell invasion in NSCLC cells.

Taken together, our results indicate that highly expressed XLOC_008466 acts as an oncogenic lncRNA that promotes the tumorigenesis and progression of NSCLC through the miR-874-MMP2/XIAP axis, indicating that XLOC_008466 could be a useful marker and potential therapeutic target for NSCLC. In our next study, we will continue to explore the potential biological effects of XLOC_008466.

\section{Acknowledgments}

The authors are grateful to all staff at the study center who contributed to this study. This study was supported by Ministry of Major Science \& Technology of Henan (201401005) and Doctoral team of the First Affiliated Hospital of Zhengzhou University.

\section{Disclosure Statement}

The authors have declared that no competing interest exists.

\section{References}

-1 Siegel RL, Miller KD, Jemal A: Cancer statistics, 2015. CA Cancer J Clin 2015;65:5-29.

2 Wang YY, Lin SX, Yang GQ, Liu HC, Sun DN, Wang YS: Clinical efficacy of CyberKnife combined with chemotherapy and hyperthermia for advanced non-small cell lung cancer. Mol Clin Oncol 2013;1:527-530.

-3 Zappa C, Mousa SA: Non-small cell lung cancer: current treatment and future advances. Transl Lung Cancer Res 2016;5:288-300.

4 Farhat FS, Houhou W: Targeted therapies in non-small cell lung carcinoma: what have we achieved so far? Ther Adv Med Oncol 2013;5:249-270.

-5 Larsen JE, Cascone T, Gerber DE, Heymach JV, Minna JD: Targeted Therapies for Lung Cancer: Clinical Experience and Novel Agents. Cancer J 2011;17:512-527.

6 Cortinovis D, Abbate M, Bidoli P, Capici S, Canova S: Targeted therapies and immunotherapy in non-smallcell lung cancer. Ecancermedicalscience 2016;10:648.

7 Isin M, Dalay N: LncRNAs and neoplasia. Clin Chim Acta 2015;444:280-288.

8 Yang JX, Rastetter RH, Wilhelm D: Non-coding RNAs: An Introduction. Adv Exp Med Biol 2016;886:13-32.

-9 Fatima R, Akhade VS, Pal D, Rao SM: Long noncoding RNAs in development and cancer: potential biomarkers and therapeutic targets. Mol Cell Ther 2015;3:5. 


\section{Cellular Physiology Cell Physiol Biochem 2017;42:126-136 \begin{tabular}{l|l} 
DOI: 10.1159/000477121 & O 2017 The Author(s). Published by S. Karger AG, Basel \\
www.karger.com/cpb
\end{tabular}}

Yang et al.: XLOC_008466 Functions as an Oncogene in NSCLC

10 Bida O, Gidoni M, Ideses D, Efroni S, Ginsberg D: A novel mitosis-associated lncRNA, MA-linc1, is required for cell cycle progression and sensitizes cancer cells to Paclitaxel. Oncotarget 2015;6:27880-27890.

11 Prensner JR, Chinnaiyan AM: The emergence of IncRNAs in cancer biology. Cancer Discov 2011;1:391-407.

12 Liz J, Esteller M: LncRNAs and microRNAs with a role in cancer development. Biochim Biophys Acta 2016;1859:169-176.

13 Li P, Li J, Yang R, Zhang F, Wang H, Chu H, Lu Y, Dun S, Wang Y, Zang W, Du Y, Chen X, Zhao G, Zhang G: Study on expression of lncRNA RGMB-AS1 and repulsive guidance molecule b in non-small cell lung cancer. Diagn Pathol 2015;10:63.

-14 Qi Y, Ooi HS, Wu J, Chen J, Zhang X, Tan S, Yu Q, Li YY, Kang Y, Li H, Xiong Z, Zhu T, Liu B, Shao Z, Zhao $\mathrm{X}$ : MALAT1 long ncRNA promotes gastric cancer metastasis by suppressing PCDH10. Oncotarget 2016;7:12693-12703.

15 Ji Q Zhang L, Liu X, Zhou L, Wang W, Han Z, Sui H, Tang Y, Wang Y, Liu N, Ren J, Hou F, Li Q: Long non-coding RNA MALAT1 promotes tumour growth and metastasis in colorectal cancer through binding to SFPQ and releasing oncogene PTBP2 from SFPQ/PTBP2 complex. Br J Cancer 2014;111:736-748.

16 Li J, Wang J, Chen Y, Li S, Jin M, Wang H, Chen Z, Yu W: LncRNA MALAT1 exerts oncogenic functions in lung adenocarcinoma by targeting miR-204.Am J Cancer Res 2016;6:1099-1107.

17 Gutschner T, Hämmerle M, Eissmann M, Hsu J, Kim Y, Hung G, Revenko A, Arun G, Stentrup M, Gross M, Zörnig M, MacLeod AR, Spector DL, Diederichs S: The non-coding RNA MALAT1 is a critical regulator of the metastasis phenotype of lung cancer cells. Cancer Res 2013;73:1180-1189.

18 Wang L, Chen Z, An L, Wang Y, Zhang Z, Guo Y, Liu C: Analysis of Long Non-Coding RNA Expression Profiles in Non-Small Cell Lung Cancer. Cell Physiol Biochem 2016;38:2389-400.

19 Tang Q, Ni Z, Cheng Z, Xu J, Yu H, Yin P: Three circulating long non-coding RNAs act as biomarkers for predicting NSCLC. Cell Physiol Biochem 2015;37:1002-1009.

-20 Qiu ZL, Shen CT, Sun ZK, Wei WJ, Zhang XY, Song HJ, Luo QY: Circulating Long Non-Coding RNAs Act as Biomarkers for Predicting 131I Uptake and Mortality in Papillary Thyroid Cancer Patients with Lung Metastases. Cell Physiol Biochem 2016;40:1377-1390.

21 Yang ZT, Li Z, Wang XG, Tan T, Yi F, Zhu H, Zhao JP, Zhou XF: Overexpression of Long Non-Coding RNA ZXF2 Promotes Lung Adenocarcinoma Progression Through c-Myc Pathway. Cell Physiol Biochem 2015;35:236070.

22 Bhan A, Mandal SS: LncRNA HOTAIR: a master regulator of chromatin dynamics and cancer. Biochim Biophys Acta 2015;1856:151-164.

23 Wang YL, Overstreet AM, Chen MS, Wang J, Zhao HJ, Ho PC, Smith M, Wang SC: Combined inhibition of EGFR and c-ABL suppresses the growth of triple-negative breast cancer growth through inhibition of HOTAIR. Oncotarget 2015;6:11150-11161.

24 Liu XH, Liu ZL, Sun M, Liu J, Wang ZX, De W: The long non-coding RNA HOTAIR indicates a poor prognosis and promotes metastasis in non-small cell lung cancer. BMC Cancer 2013;13:464.

25 Vennin C, Spruyt N, Dahmani F, Julien S, Bertucci F, Finetti P, Chassat T, Bourette RP, Le Bourhis X, Adriaenssens E: H19 non coding RNA-derived miR-675 enhances tumorigenesis and metastasis of breast cancer cells by downregulating c-Cbl and Cbl-b. Oncotarget 2015;6:29209-29223.

-26 Xing CY, Hu XQ Xie FY, Yu ZJ, Li HY, Bin-Zhou, Wu JB, Tang LY, Gao SM: Long non-coding RNA HOTAIR modulates c-KIT expression through sponging miR-193a in acute myeloid leukemia. FEBS Lett 2015;589:1981-1987.

27 Kesanakurti D, Maddirela DR, Chittivelu S, Rao JS, Chetty C: Suppression of tumor cell invasiveness and in vivo tumor growth by microRNA-874 in non-small cell lung cancer. Biochem Biophys Res Commun 2013;434:627-633.

28 Wang H, Zhu Y, Zhao M, Wu C, Zhang P, Tang L, Zhang H, Chen X, Yang Y, Liu G: miRNA-29c Suppresses Lung Cancer Cell Adhesion to Extracellular Matrix and Metastasis by Targeting Integrin $\beta 1$ and Matrix Metalloproteinase2 (MMP2). PLoS One 2013;8:e70192.

29 Qin S, Yang C, Zhang B, Li X, Sun X, Li G, Zhang J, Xiao G, Gao X, Huang G, Wang P, Ren H: XIAP inhibits mature Smac-induced apoptosis by degrading it through ubiquitination in NSCLC. Int J Oncol 2016;49:1289-1296. 